\title{
Electronic Speckle Pattern Shearing Interferometry using Photopolymer Diffractive Optical Elements for Vibration \\ Measurements
}

\author{
Emilia Mihaylova \\ Technological University Dublin, emilia.mihaylova@tudublin.ie \\ Izabela Naydenova \\ Technological University Dublin, izabela.naydenova@tudublin.ie \\ Suzanne Martin \\ Technological University Dublin, suzanne.martin@tudublin.ie
}

See next page for additional authors

Follow this and additional works at: https://arrow.tudublin.ie/cieocon2

Part of the Engineering Physics Commons, and the Optics Commons

\section{Recommended Citation \\ Mihaylova, E. et al. (2005) Electronic Speckle Pattern Shearing Interferometry Using Photopolymer Diffractive Optical Elementsfor Vibration Measurements. Proceedings of 6th international conference on Vibration Measurements by Laser Techniques: Advance Applications, Ancona, Italy, pp.73-78, 2004. doi.org/10.21427/bf60-qw60}

This Conference Paper is brought to you for free and open access by the Centre for Industrial and Engineering Optics at ARROW@TU Dublin. It has been accepted for inclusion in Conference Papers by an authorized administrator of ARROW@TU Dublin. For more information, please contact arrow.admin@tudublin.ie, aisling.coyne@tudublin.ie,gerard.connolly@tudublin.ie.

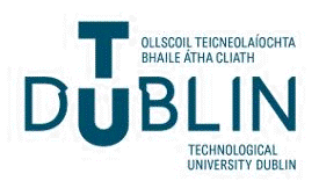




\section{Authors}

Emilia Mihaylova, Izabela Naydenova, Suzanne Martin, and Vincent Toal

This conference paper is available at ARROW@TU Dublin: https://arrow.tudublin.ie/cieocon2/29 
2004-01-01

\section{Electronic Speckle Pattern Shearing Interferometry Using Photopolymer Diffractive Optical Elementsfor Vibration Measurements}

Emilia Mihaylova

Dublin Institute of Technology, emilia.mihaylova@dit.ie

Izabela Naydenova

Dublin Institute of Technology, izabela.naydenova@dit.ie

Suzanne Martin

Dublin Institute of Technology, suzanne.martin@dit.ie

Vincent Toal

Dublin Institute of Technology, vincent.toal@dit.ie

\section{Recommended Citation}

E. Mihaylova, I. Naydenova, S. Martin, V. Toal, Electronic Speckle Pattern Shearing Interferometry Using Photopolymer Diffractive Optical Elementsfor Vibration Measurements, Proceedings of 6-th international conference on Vibration Measurements by Laser Techniques: Advance Applications, Ancona, Italy, pp.73-78, 2004. 


\title{
Electronic Speckle Pattern Shearing Interferometry Using Photopolymer Diffractive Optical Elements for Vibration Measurements
}

\author{
Emilia Mihaylova, Izabela Naydenova, Suzanne Martin, Vincent Toal \\ Centre for Industrial and Engineering Optics \\ Dublin Institute of Technology, Kevin Street, Dublin 8, Ireland \\ e-mail: emilia.mihaylova@ dit.ie
}

\begin{abstract}
Electronic speckle pattern shearing interferometry (ESPSI) is superior to Electronic speckle pattern interferometry (ESPI) when strain distribution, arising from object deformation or vibration, need to be measured. This is because shearography provides data directly related to the spatial derivatives of the displacement. Further development of ESPSI systems could be beneficial for wider application to the measurement of mechanical characteristics of vibrating objects.

Two electronic speckle pattern shearing interferometers (ESPSI) suitable for vibration measurements are presented. In both ESPSI systems photopolymer holographic gratings are used to shear the images and to control the size of the shear. The holographic gratings are recorded using an acrylamide-based photopolymer material. Since the polymerisation process occurs during recording, the holograms are produced without any development or processing. The ESPSI systems with photopolymer holographic gratings are simple and compact. Introducing photopolymer holographic gratings in ESPSI gives the advantage of using high aperture optical elements at relatively low cost.

It is demonstrated that both ESPSI system can be used for vibration measurements. The results obtained are promising for future applications of the systems for modal analysis.
\end{abstract}

KEYWORDS: ESPSI, shearing interferometry, holographic gratings, modal analysis, vibrations

\section{INTRODUCTION}

Electronic speckle pattern shearing interferometry (ESPSI) enables direct measurements of displacement derivatives to be made with variable sensitivity ${ }^{1-8}$. A common approach to generate two sheared images of the object in ESPSI systems is to use a Michelson interferometric optical set-up. The shear is introduced and controlled by tilting one of the mirrors.

The idea of using a holographic grating for shearing of the two images in speckle shearing interferometry is not new. Some early non-electronic systems used photographic gratings ${ }^{5-7}$, but ESPSI using gratings to shear the image enables observation of real-time fringe formation ${ }^{8,9}$. Recently there has been an increasing interest in the application of shearography for modal analysis of vibrating objects. New interferometric systems are of interest for engineering and industrial applications.

We suggest new applications of photopolymer holographic gratings in two ESPSI optical set-ups. Self-processing acrylamide based photopolymer ${ }^{10}$ is used as a recording medium for recording holographic gratings. The optimized photopolymer material gives good diffraction efficiencies up to $94 \%$ for an exposure of $80 \mathrm{~mJ} / \mathrm{cm}^{2}$ and it performs well in the transmission mode of hologram recording. In both ESPSI systems the photopolymer gratings are used to shear the image. 


\section{THEORY}

When two light waves interfere, the following equation ${ }^{11}$ relates their relative phase $\Phi$ at a location to their relative geometrical path length $L$ :

$$
\Phi=\frac{2 \pi}{\lambda} n L-\beta
$$

where $\lambda$ is the wavelength of the laser light, $n$ is the refractive index of the medium through which the laser light is transmitted, and $\beta$ is a constant phase. The change in the relative phase $\Delta=\delta \Phi$ or phase change, which manifests as visible fringes, can be effected by an incremental change in any of the three parameters $\lambda, n$, and $L$. Thus,

$$
\Delta=\frac{\partial \Phi}{\partial \lambda} \delta \lambda+\frac{\partial \Phi}{\partial n} \delta n+\frac{\partial \Phi}{\partial L} \delta L=-\frac{2 \pi L n}{\lambda^{2}} \delta \lambda+\frac{2 \pi L}{\lambda} \delta n+\frac{2 \pi n}{\lambda} \delta L
$$

where $\delta \lambda, \delta n$, and $\delta L$ denote respectively, the incremental change in wavelength, in refractive index, and in relative geometrical path length of the interfering waves.

If the same wavelength is used and the test environment is still air $(n=1)$, only the $\delta L$ term in Eq. (2) is nonzero, resulting in the following equation for the phase change ${ }^{12}$ :

$$
\Delta=\frac{2 \pi}{\lambda}[A \delta u+B \delta v+C \delta w]
$$

where $u, v$ and $w$ are the displacement components of the neighboring point $P^{\prime}(x+\Delta x, y, z+\Delta z)$ relative to point $P(x, y, z)$ on the test surface, and $A, B$, and $C$ are sensitivity factors that are related to the optical arrangement. For small image shearing $\Delta x$, the displacement terms in Eq. (3) can be expressed in terms of partial derivatives:

$$
\Delta=\frac{2 \pi}{\lambda}\left[A \frac{\partial u}{\partial x}+B \frac{\partial v}{\partial x}+C \frac{\partial w}{\partial x}\right]
$$

In our case (Fig. 1 and Fig. 2) the object beam lies in the (x, z) plane so there is no sensitivity in the y direction.. The phase change is:

$$
\Delta=\frac{2 \pi}{\lambda}\left[A \frac{\partial u}{\partial x}+C \frac{\partial w}{\partial x}\right]
$$

Consider the situation of an ESPSI system with one holographic grating in front of the CCD camera and small image shear $\Delta x$. The phase difference $\Delta$ can be expressed as ${ }^{8}$

$$
\Delta=\frac{2 \pi}{\lambda}\left[\frac{\partial u}{\partial x} \sin \theta+\frac{\partial w}{\partial x}(1+\cos \theta)\right] \Delta x
$$

The dark fringes correspond to $\Delta=2 n \pi$, where $n$ is the fringe order. In this case:

$$
\frac{\partial u}{\partial x} \sin \theta+\frac{\partial w}{\partial x}(1+\cos \theta)=\frac{\lambda n}{\Delta x}
$$




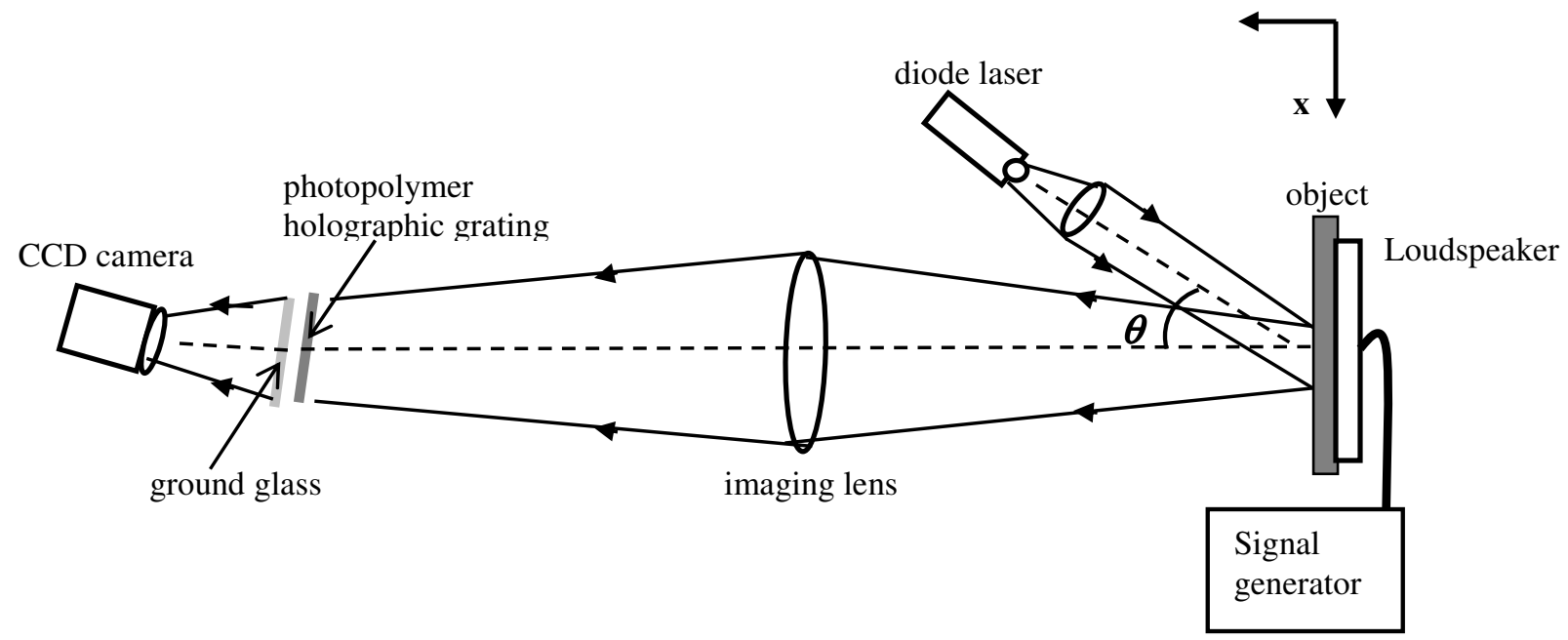

Fig.1. ESPSI system with one photopolymer grating

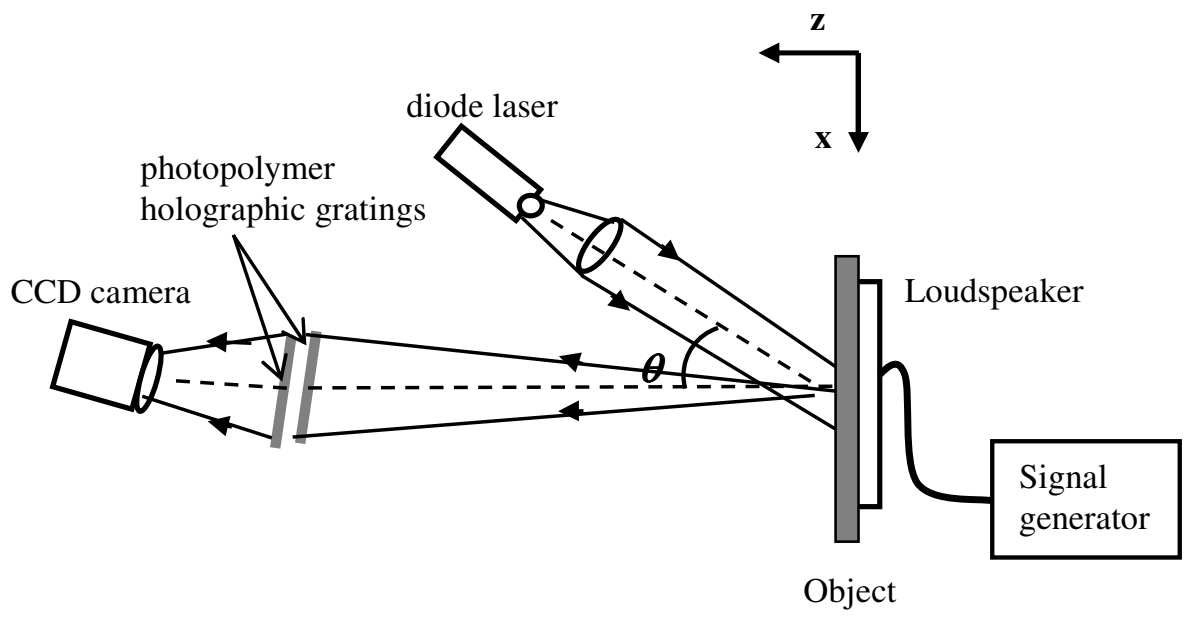

Fig.2. ESPSI system with two photopolymer gratings 


\section{EXPERIMENT}

Holographic gratings with spatial frequency of 500 lines per mm were recorded using the second harmonic of a CW Nd$\mathrm{YVO}_{4}$ laser $(\lambda=532 \mathrm{~nm})$. Recording time and intensity were $20 \mathrm{~s}$ and $3.5 \mathrm{~mW} / \mathrm{cm}^{2}$ respectively. The diameter of the gratings is $40 \mathrm{~mm}$. Diffraction efficiency in the +1 order is $60 \%$.

A Helium-Neon laser, with wavelength $633 \mathrm{~nm}$ and output power of $20 \mathrm{~mW}$, is used as the light source. In both set-ups the laser beam illuminates the object at an angle $\theta=30^{\circ}$ to the normal to the object surface.

The scheme of the ESPSI system with a single photopolymer holographic grating is presented in Figure 1. A lens images the object onto a ground glass, which acts as a diffusing screen. A holographic photopolymer diffraction grating is placed in front of the ground glass. The intensities of the zero and the first order of diffraction were equalized by rotation of the grating. The rotation of the grating leads to slight off-Bragg angular adjustment and decrease in the intensity of the first order thus offering the possibility for fine adjustment of both image and sheared image intensities.

The arrangement of the electronic speckle pattern shearing interferometric system with two photopolymer holographic gratings is similar to the first one but simpler (Fig. 2). The shearing element consists of two holographic gratings. In the shearography system with two holographic gratings (Fig. 3) two pairs of beams can be used - $(00,11)$ or $(10,01)$. The intensities for the beams in each of the pairs can be equalized by rotation of the gratings after which the CCD camera is realigned.

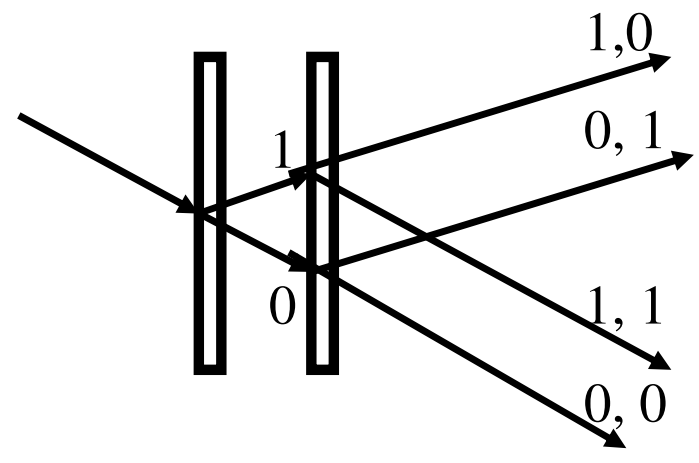

Fig. 3. Propagation of the beams through the shear introducing diffraction gratings.

Both ESPSI system were used for studying the vibration modes of an edge clamped aluminium diaphragm $(75 \mathrm{~mm}$ diameter, $0.5 \mathrm{~mm}$ thick). The diaphragm was excited at different frequencies in the range from $200 \mathrm{~Hz}$ to $7000 \mathrm{~Hz}$ by a loudspeaker placed behind it. The loudspeaker is driven by a sinusoidal signal of amplitude $0.5 \mathrm{~V}$ to $1 \mathrm{~V}$. Time-averaged TV shearography was used for all vibration studies.

\section{RESULTS AND DISCUSSION}

The two ESPSI systems were used to record different vibrating modes of the an edge clamped aluminium diaphragm (Fig. 4 - 6). A filter with a $3 \times 3$ window was used to reduce the speckle noise in the images.

As can be seen from the Fig 4 and 5 increase of the shear leads to higher sensitivity and lower amplitude of vibration is required in order to obtain the same contrast of the characteristic interference pattern.

An important factor for the performance of the ESPSI system with two holographic gratings is the angular selectivity of the shear-introducing components. This requires careful adjustment of the spatial frequency and the thickness of the photopolymer gratings in order to decrease their angular selectivity. 


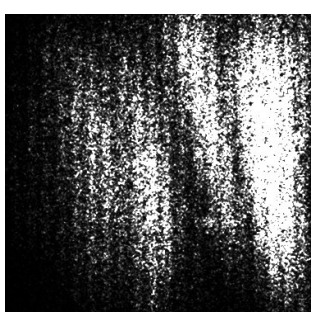

$2100 \mathrm{~Hz}$

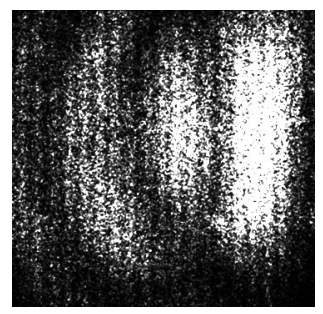

$3800 \mathrm{~Hz}$

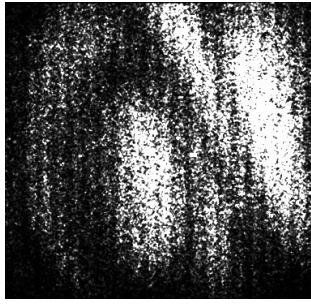

$5200 \mathrm{~Hz}$

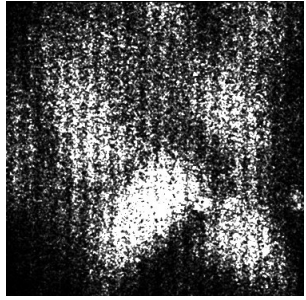

$6200 \mathrm{~Hz}$

Fig. 4. ESPSI vibration modes of the central part of an edge clamped aluminium diaphragm recorded with the first system. The shear is $8 \mathrm{~mm}$. The amplitude of the sinusoidal signal driving the loudspeaker is $1 \mathrm{~V}$. The field of view is $23 \mathrm{~mm} \times 23 \mathrm{~mm}$

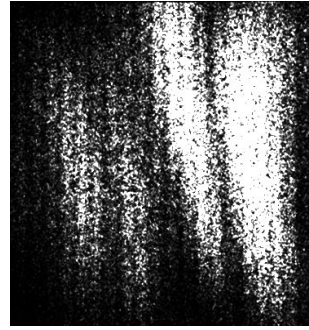

$2100 \mathrm{~Hz}$

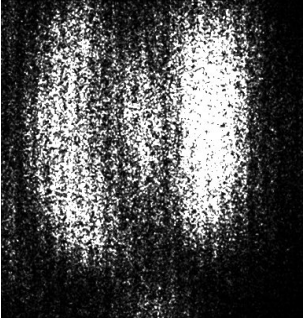

$3800 \mathrm{~Hz}$

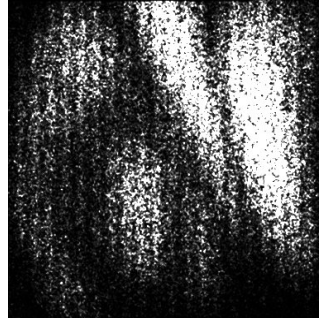

$5200 \mathrm{~Hz}$

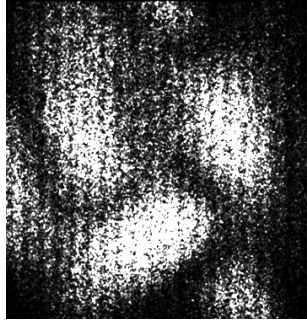

$6200 \mathrm{~Hz}$

Fig. 5. ESPSI vibration modes of the central part of an edge clamped aluminium diaphragm recorded with the first system. The shear is $11 \mathrm{~mm}$. The amplitude of the sinusoidal signal driving the loudspeaker is $0.5 \mathrm{~V}$. The field of view is $23 \mathrm{~mm} \times 23 \mathrm{~mm}$

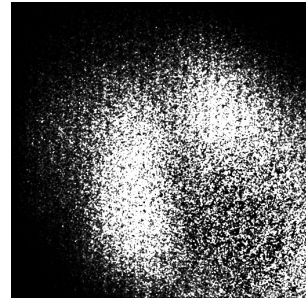

$2100 \mathrm{~Hz}$

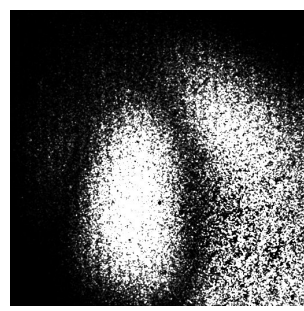

$3800 \mathrm{~Hz}$

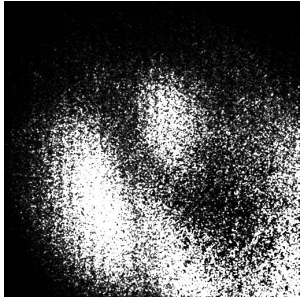

$5200 \mathrm{~Hz}$

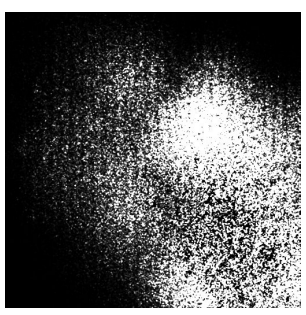

$6200 \mathrm{~Hz}$

Fig. 6. ESPSI vibration modes of the central part of an edge clamped aluminium diaphragm recorded with the second system. The shear is $8 \mathrm{~mm}$. The amplitude of the sinusoidal signal driving the loudspeaker is $0.5 \mathrm{~V}$. The field of view is $30 \mathrm{~mm} \times 30 \mathrm{~mm}$ 


\section{CONCLUSIONS}

The ESPSI systems using diffraction gratings to shear the image are simple and flexible and offer a simple way to introduce discrete shear steps between two images. An additional advantage is the low cost of such a system. The contrast of the time averaged vibration fringes recorded with the first system is above $90 \%$. It is demonstrated that both ESPSI system can be used for vibration measurements. The results obtained are promising for future applications of the systems for modal analysis.

\section{ACKNOWLEDGMENTS}

Acknowledgements are made to Technological Sector Research Programme Strand III supported by the Irish Government. Emilia Mihaylova and Izabela Naydenova would like to thank the Arnold F. Graves Scholar Programme and FOCAS at Dublin Institute of Technology.

\section{REFERENCES}

1. C. Joenathan C. and Torroba R., "Simple electronic speckle shearing pattern interferometer", Opt. Lett. 15 (20), 1159-1161 (1990).

2. R. S. Sirohi, "Speckle methods in experimental mechanics", in Speckle Metrology, Ed. R. S. Sirohi, Mercel Dekker, New York (1993).

3. Y. M. He, C. J. Tay, H. M. Shang, "Digital phase-shifting shearography for slope measurement", Opt. Eng. 38 (9), 1586-1590 (1999).

4. H. M. Shang, Y. Y. Hung, W. D. Luo, F. Chen, "Surface profiling using shearography", Opt. Eng. 39(1), 23-31 (2000).

5. P. Hariharan, "Speckle-shearing interferometry: a simple optical system", Appl. Opt. 14 (11), 2563 (1975).

6. Y. Iwahashi, K. Iwata, and R. Nagata, "Single-aperture speckle shearing interferometry with a single grating", Appl. Opt. 23 (2), 247-249 (1984).

7. C. Joenathan and R. S. Sirohi, "Holographic gratings in speckle shearing interferometry", Appl. Opt. 24 (17), 27502751 (1985).

8. H. Rabal, R. Henao, R. Torroba, "Digital speckle pattern shearing interferometry using diffraction gratings", Optics Comm. 126, 191-196 (1996).

9. C. Joenathan, L. Bürkle, "Elecktronic speckle pattern shearing interferometer using holographic gratings", Opt. Eng. 36 (9), 2473-2477 (1997).

10. S. Martin, P. Leclère, V. Toal, Y. Renotte and Y. Lion, "Characterisation of acrylamide-based photopolymer holographic recording material", Optical Engineering, 32 (12), 3942 - 3946 (1994).

11. C. M. Vest, Holographic Interferometry (John Wiley, New York 1979).

12. Y. Y. Hung and C. Y. Liang, "Image shearing camera for direct measurement of surface-strains", Appl. Opt. 10(7), 1046-1050 (1979). 